Article available at nttp://www.parasite-journal.org or nttp://dx.dol.org/10.1051/parasite/2000074275

\title{
EXPERIMENTAL INFECTION OF PIGS WITH THREe DOSE LEVELS OF TRICHURIS SUIS
}

\author{
PEDERSEN S.* \& SAEED I.*
}

Summary :

The objective of the study was to follow the course of Trichuris suis infection in pigs given infective eggs at low (400 eggs), medium (4,000 eggs) and high inoculation dose (40,000 eggs), respectively. Interestingly, despite a 100-fold difference in dose level no significant difference was found in either blood parameters, total faecal egg excretion, fecundity or worm burdens at necropsy 12 weeks post inoculation. The highest and lowest median faecal egg output was found in the medium and high dose group, respectively. With increasing dose level, worm size, establishment and prevalence of $T$. suis positive pigs decreased while worms were dislocated aborally. In addition there was a highly significant correlation between female worm burden and faecal egg excretion.

KEY WORDS : Trichuris suis, whipworm, pig, single infection, dose.
Résumé : INFECTION EXPÉRIMENTALE DE PORCS À L'AIDE DE TROIS DOSES DIFFÉRENTES DE TRICHURIS SUIS

L'objectif de l'étude était de suivre le cours d'une infestation par Trichuris suis chez des porcs à qui ont été donnés des œuts embryonnés à des doses différentes de 400, 4000 et 40000 œufs respectivement. Malgré une différence de un à cent du niveau d'infestation, aucune différence marquante n'a été trouvée, ni dans les paramètres sanguins, ni dans les excrétions fécales, ni dans la fécondité, ni dans le niveau de l'infestation à l'examen nécropsique, 12 semaines après l'inoculation. C'est dans les groupes ayant reçu les doses moyennes et fortes que sont trouvées respectivement les excrétions d'œufs les plus hautes et les plus basses. Quand la dose infestante a été augmentée, la taille des vers, le nombre de trichures par porc et la prévalence d'infestation ont baissé, tandis que les vers ont été rejetés per os. De plus, il y avait une relation significative entre le nombre de vers femelles et le niveau d'excrétions fécales.

MOTS CLÉS : Trichuris suis, trichure, porc, infection unique, dose.

tive anatomy and physiology (Miller \& Ullrey, 1987). Furthermore, T. suis is very similar to the human whipworm T. trichiura with respect to life cycle, morphology, location and interaction with the intestinal mucosa (Holland, 1987). Therefore, experimental T. suis infection in the pig is considered to be a good model for direct comparison with the equivalent infection in humans, which affects 500 million people worldwide (Stephenson, 1987).

Hitherto, most experimental studies have employed high inoculation doses, i.e. 15,000-400,000 infective eggs (Powers et al., 1960; Beer et al., 1971; Batte \& Moncol, 1972; Beer \& Lean, 1973; Beer et al., 1973; Batte et al., 1977; Hale \& Stewart, 1979). However, under natural conditions ingestion of smaller infective doses is more likely to occur, partly causing less intense activation of the host immune response, partly modulating the population dynamics of Trichuris. Eventually, a more prolonged and chronic infestation could result as documented in the clinical syndrome of children (Holland, 1987). Few experimental studies have been performed to investigate the course of infection after patency following inoculation at different dose levels. In the present study, three inoculation doses were used: 1) a low dose imitating a natural low level of infection; 2) a medium dose not expected

\footnotetext{
* Danish Centre for Experimental Parasitology, Royal Veterinary and Agricultural University, Ridebanevej 3, DK-1870 Frederiksberg C, Copenhagen, Denmark.

Correspondence: Søren Pedersen.

Tel.: + 4535282775 - Fax: + 4535282774

E-mail: skp@kvl.dk
} 
to produce clinical signs or crowded worm populations (Johansen et al., 1997) and 3) a high dose known to be able to provoke clinical symptoms (Powers et al., 1960). Single doses given to parasite-naïve pigs were chosen, which made it possible to investigate the result of the intricate interaction between the initial immune response and the entire development from larvae to adults of one age-group of parasites, without introducing an interfering effect of acquired resistance associated with trickle infections.

The aim of our study was to follow the course of T. suis infection in pigs given infective eggs at low (400 eggs), medium (4,000 eggs) and high inoculation doses (40,000 eggs), respectively. During the course of infection clinical, haematological and coprological parameters were monitored, and T. suis burdens were determined at autopsy 12 weeks post inoculation (p.i.).

\section{MATERIALS AND METHODS}

\section{ANIMALS AND FEEDING}

T Twenty-nine specific-pathogen-free cross-bred Danish Landrace/ Yorkshire/ Duroc pigs of both sexes weighing $77 \pm 15.5 \mathrm{~kg}$ (mean \pm S.D.) were purchased from a helminth-free experimental farm and housed together in a stable $\left(5 \times 25 \mathrm{~m}^{2}\right)$ with straw bedding and access to pasture via a soiled area. The pigs had free access to water via drinking nipples. The pigs were weighed every second week.

A standard diet consisting of ground barley plus a commercial premix (16\% crude protein) was fed to the pigs

\section{EXPERIMENTAL DESIGN}

The pigs were randomly divided into four groups; an uninoculated control group (four females and two males), a low dose group (400 eggs per pig; six females and two males), a medium dose group (4,000 eggs per pig; five females and three males) and a high dose group (40,000 eggs per pig; four females and three males).

Infective T. suis eggs were originally isolated in 1996 from soil in a small organic farm and subsequently passaged in helminth-naïve pigs. The eggs used in the present experiment were isolated from faeces of pigs, embryonated in vermiculite according to the method described by Burden \& Hammet (1976) and subsequently stored at $10^{\circ} \mathrm{C}$ for two years. The pigs were inoculated orally via a stomach tube. Faecal samples were collected from the rectum of each pig two, four and six weeks (wks) p.i. and weekly hereafter. Egg counts were evaluated using a concentration McMaster Technique as described by Roepstorff \& Nansen (1999), with a detection limit of 20 eggs per gram (e.p.g.). Blood samples were taken biweekly from the truncus bijugularis of each pig and haemoglobin concentration, packed cell volume (PCV), serum protein concentration, serum albumin concentration, albumin/globulin $(\mathrm{A} / \mathrm{G})$ ratio and the peripheral eosinophil count were measured and analysed.

\section{NECROPSY AND WORM RECOVERY}

All pigs were slaughtered 12 wks p.i. Feed was withheld on the day of necropsy and pigs were euthanised by electrical stunning followed by exsanguination. Trichuris suis worms were recovered according to the method described by Roepstorff \& Murrell (1997). The large intestine was divided into five sections, designated as follows, starting at the caecum: 1: caecum; 2: $0-20 \%$ of the total length of colon; 3: $21-40 \%$; $4: 41-$ $60 \%$ and 5: 61-100\% including rectum. The sections were opened with scissors and the intestinal wall was gently washed to liberate T. suis. Representative aliquots of $10 \%$ of the intestinal contents and intestinal wall washings were washed over a sieve of mesh size $212 \mu \mathrm{m}$. Retained samples were fixed in $15^{\circ} \mathrm{C}$ iodine (80 $\mathrm{g}$ iodine and $400 \mathrm{~g}$ potassium iodide in $800 \mathrm{ml}$ of distilled water) for later isolation of T. suis. Samples were transferred to a Petri dish held over a light table and decolourized with a $30 \%$ sodium thiosulphate solution. Worms visible with the naked eye were recovered, stored in $70 \%$ ethanol and differentiated according to developmental stage and sex (Beer, 1973a). The intestinal location of T. suis in each pig was estimated by assuming that all worms in each section were localized in the middle of that particular section. The percentage of worms in each section of individual pigs was calculated, allowing relative distributions of T. suis in sections of the large intestine to be compared between groups. Body lengths of intact worms were measured by means of a stereo microscope and a digital image analysis system (Microvision ${ }^{\circledR}$, DTI, Denmark). For those pigs harbouring more than 10 worms of each sex, 10 female and 10 male worms were selected as randomly as possible. The proportion of female worms was calculated by dividing the number of female worms by the total number of adult worms. Percentage establishment was defined as the percentage of the infective eggs recovered as worms. For those pigs harbouring female worms, fecundity was estimated by dividing e.p.g. at the day of slaughter by the number of females recovered.

\section{STATISTICAL ANALYSIS}

Worm numbers, intestinal location, worm distribution in sections, percentage establishment, proportion of female T. suis, worm length, faecal egg counts, worm 
fecundity and body weight were compared using oneway analysis of variance (ANOVA). Where appropriate the non-parametric Kruskal-Wallis test was used.

Univariate repeated measurements ANOVA was used for longitudinal analyses for body weight and blood parameters. Longitudinal analysis of faecal egg excretion was performed by estimating the area under the e.p.g. curve of individual pigs from six to 12 wks p.i. It was assumed that the counts obtained weekly represented mean daily counts for the whole week. Groups were then compared using the Kruskal-Wallis test.

For those pigs harbouring female T. suis at slaughter Pearson's correlation coefficient was used to relate the female worm burden to faecal egg excretion. Before analysis worm numbers and e.p.g. were logarithmically transformed $\left(\log _{10}(\mathrm{x}+10)\right)$. The prevalences of T. suis positive pigs at slaughter were compared between groups using Fisher's Exact test. The parameter of the negative binomial distribution, $k$, was estimated as $k=$ mean $^{2} /$ (variance-mean); $k$ decreases as overdispersion increases. Mean and variance were calculated on total worm burdens.

\section{RESULTS}

\section{WORM RECOVERY}

The number of T. suis worms recovered are presented in Table I. Two pigs from the control group picked up infection of 10 and 90 T. suis, respectively. Mean $( \pm$ S.E.M.) worm numbers were $93 \pm 39,399 \pm 280$ and $3,536 \pm 2,291$ in the low, medium and high dose group,

\begin{tabular}{lrrrrrr}
\hline & \multicolumn{5}{c}{ Intestinal section } \\
\cline { 2 - 6 } Group & $\mathrm{S} 1$ & $\mathrm{~S} 2$ & $\mathrm{~S} 3$ & $\mathrm{~S} 4$ & $\mathrm{~S} 5$ & Total \\
\hline Control & 80 & 10 & 0 & 0 & 0 & 90 \\
$(n=6)$ & 10 & 0 & 0 & 0 & 0 & 10 \\
Low & 30 & 140 & 70 & 0 & 10 & 250 \\
$(n=7)$ & 100 & 110 & 0 & 0 & 10 & 220 \\
& 20 & 20 & 50 & 0 & 0 & 90 \\
& 0 & 0 & 60 & 0 & 0 & 60 \\
& 0 & 0 & 20 & 0 & 0 & 20 \\
Medium & 0 & 0 & 10 & 0 & 0 & 10 \\
$(n=8)$ & 340 & 1,140 & 370 & 410 & 0 & 2,260 \\
& 30 & 150 & 490 & 50 & 10 & 730 \\
& 20 & 50 & 40 & 60 & 10 & 180 \\
High & 10 & 0 & 0 & 0 & 0 & 10 \\
$(n=7)$ & 0 & 0 & 0 & 0 & 10 & 10 \\
\hline & 1,820 & 1,860 & 4,790 & 4,130 & 700 & 13,300 \\
& 1,590 & 3,150 & 2,070 & 3,440 & 1,200 & 11,450 \\
\hline
\end{tabular}

Table I. - Number of Trichuris suis recovered from the large intestine of pigs inoculated with $400,4,000$ or 40,000 eggs, together with uninoculated control pigs. Only pigs harbouring T. suis at slaughter are included. S1: section 1 (caecum). S2: section $2(0-20 \%$ of the total length of colon). S3: section 3 (21-40\%). S4: section 4 (41$60 \%)$. S5: section 5 (61-100 \% including rectum). Total: all five sections. respectively, representing a mean (maximum) individual establishment of 23 (63) \%, 10 (57) \% and 9 (33) \%, respectively (Fig. 1). Neither the number of worms $(P=$ $0.57)$ nor establishment of infection $(P=0.17)$ were significantly different between experimental groups. Six pigs ( $86 \%)$ in the low dose group, five pigs $(63 \%)$ in the medium dose group and two pigs (29\%) in the
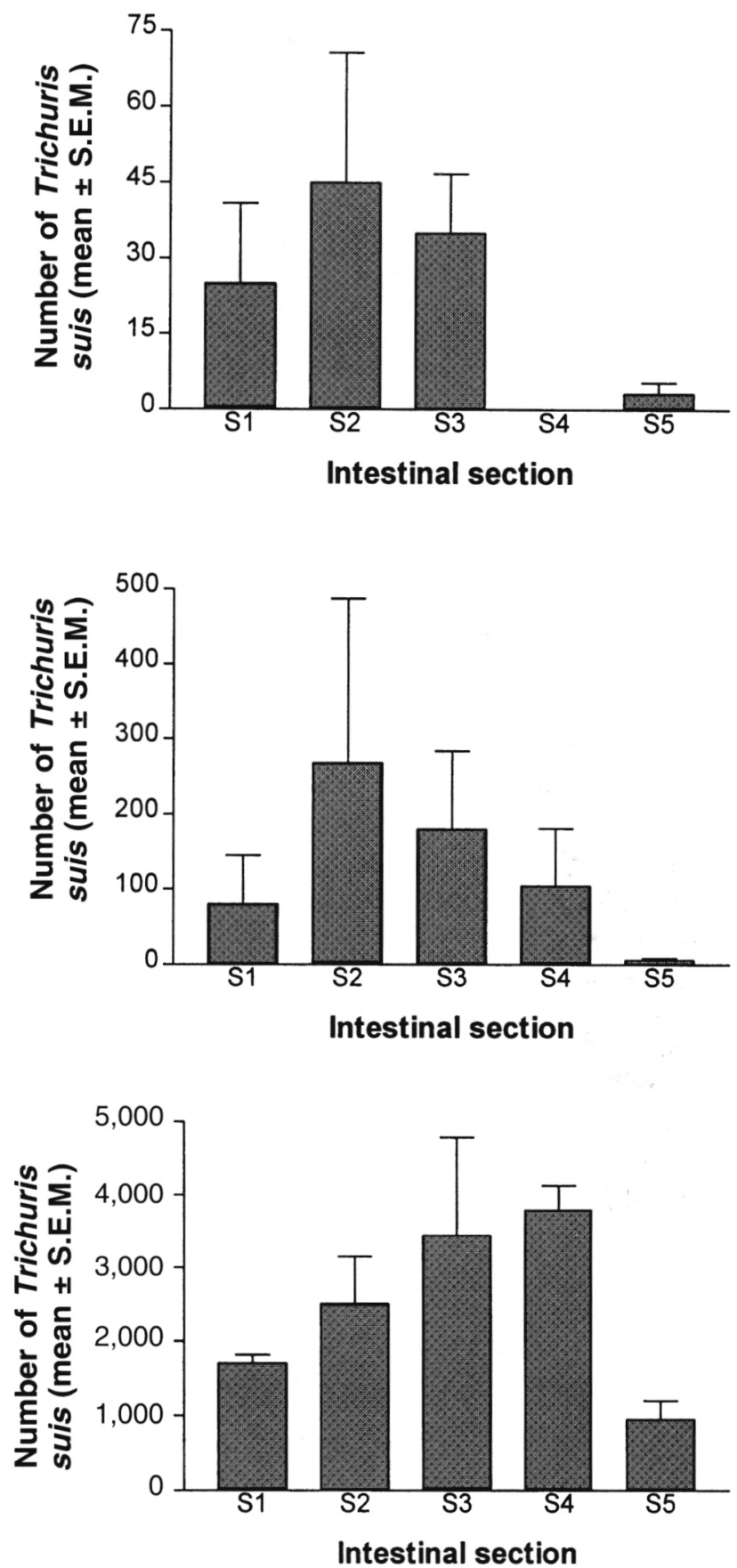

Fig.1. - Mean ( \pm S.E.M.) number of $T$. suis recovered from five intestinal sections of the A) low, B) medium and C) high dose group, respectively. S1: caecum. S2: $0-20 \%$ of the total length of colon. S3: $21-40 \%$. S4: 41-60\%. S5: 61-100\% including rectum. Only T. suis positive pigs are included. 


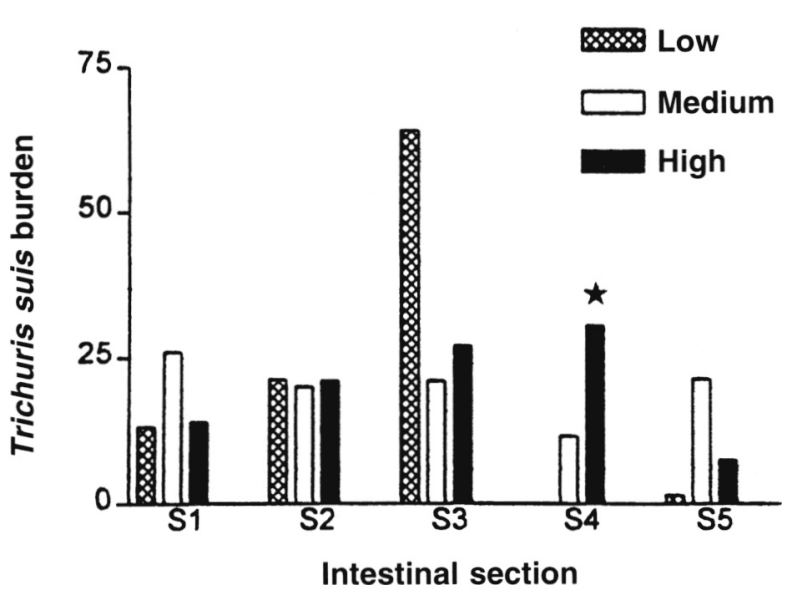

Fig.2. - Comparison of the relative distribution (\%) of T. suis in the low, medium and high dose groups. S1: caecum. S2: 0- $20 \%$ of the total length of colon. S3: 21-40 \%. S4: 41-60\%. S5: 61-100\% including rectum. The asterisk marks the significantly higher percentage of the worm burden in $\mathrm{S} 4$ found in the high compared to the low dose group $(P=0.026)$.

high dose group harboured worms but this was not significant $(P>0.10)$. In the high dose group a significantly higher percentage of the worm burden was recovered from section 4 compared with the low dose group $(P=0.026)$. Median intestinal location of the worm burden was 2.6, 2.8 and 3.0 for the low, medium and high dose group, respectively $(P=0.47)$. The relative distributions of worms in the five sections of the large intestine for the three groups are shown in Fig. 2. Only adult $T$. suis were recovered and they ranged from 16 to $48 \mathrm{~mm}$ in length. Total median lengths of males and females as well as the total median (min.max.) worm lengths (low: 33.9 (28.8-40.2), medium: 36.1 (28.3-40.9), high: $26.7(25.8-27.5) ; P=0.10)$ did not differ significantly. The median proportion of female $T$. suis was not significantly different between groups (control: 0.78; low: 0.41; medium: 0.43; high: $0.43 ; P=0.90)$. The parameter $k$ of the negative binomial distribution was found to be $k<1$ in all three experimental groups (low: 0.83; medium: 0.25; high: 0.34) indicating a high degree of aggregation of the adult $T$. suis populations.

Eight wks p.i. one pig from the high dose group - which harboured worms at necropsy - excreted adult T. suis worms in the faeces.

\section{FAECAL EGG COUNTS}

Faecal egg excretion in some of the inoculated pigs started six wks p.i. There were one and four pigs in the low and high dose group, respectively, which did not have faecal egg counts above 20 e.p.g. at any occasion. The highest and lowest mean e.p.g. levels were observed in the the high and low dose group, res- pectively, and all groups peaked nine wks p.i. (low: 1,026; medium: 5,580; high: 24,643 e.p.g.) (Fig. 3A). Figure $3 \mathrm{~B}$ shows that between wk seven and 12 the median was 140-580 ( $\max . w k 10), 10-3,725$ ( $\max$. wk seven) and 0-20 (max. wk 7-8) e.p.g. for the low, medium and high dose group, respectively, reflecting the decrease in number of pigs excreting eggs with increasing inoculation dose. In spite of these observations, longitudinal analysis showed that there was no significant difference in total median (min.-max.) egg excretion between experimental groups during the study i.e. low: 2,740 (20-14,120), medium: 8,670 (42073,520) and high: $40(20-289,680)$ e.p.g. $(P=0.36)$. Faecal egg excretion did not differ between groups at any sampling time $(P>0.13)$.

Each female T. suis excreted a median (min.-max.) of 9.5 (3.4-160), $0.4(0-6)$ or $9.1(8.6-9.5)$ e.p.g. in the low, medium and high dose group, respectively. Comparison of the median fecundity revealed no significant difference between groups $(P=0.063)$.

For pigs harbouring female $T$. suis there was a significant correlation between female worms recovered at

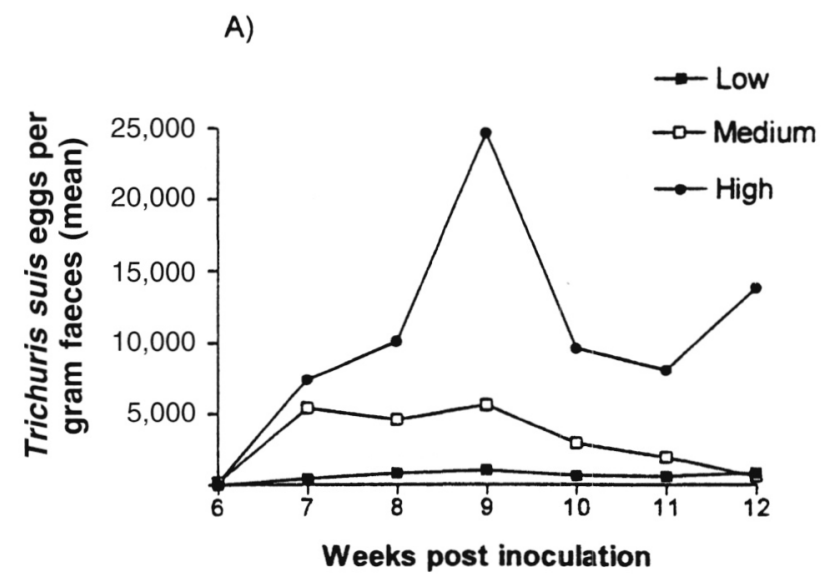

B)

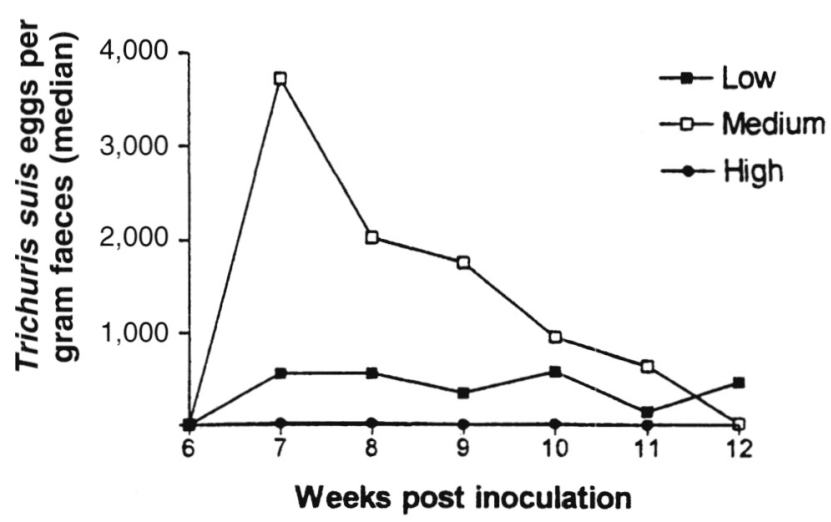

Fig.3. - A) Mean and B) median T. suis eggs per gram faeces (e.p.g.) in the low, medium and high dose groups from six to 12 weeks post inoculation. 
necropsy and concurrent e.p.g. $(r=0.80, n=12, P=$ 0.002). Two pigs did not excrete eggs but harboured worms (false-negative egg counts) while three pigs showed false-positive faecal egg counts (20, 20 and 120 e.p.g.) at slaughter.

\section{HaEMATOlOGY \& ANIMAL PERFORMANCE}

Longitudinal analysis of serum protein $(P=0.43)$ and albumin levels $(P=0.63), \mathrm{A} / \mathrm{G}$ ratio $(P=0.63)$, haemoglobin $(P=0.09)$, PCV $(P=0.11)$ and peripheral eosinophil counts $(P=0.84)$ did not reveal any significant differences between controls and/or inoculated groups. However, the high dose group experienced a significantly higher decrease in albumin levels between wk 2-6 p.i. $(P=0.012)$.

During the study there were no significant differences in body weight gains between groups at any time. Overall, there was no difference between groups in weight gains with time $(P=0.71)$. One female pig in the high dose group developed diarrhoea seven wks p.i. and became emaciated. Looseness of the faeces occasionally occurred in a number of infected pigs in the medium and high dose groups. During the study one female pig from the low dose group died from incarcerated umbilical hernia. Another pig in the low dose group had a diseased leg and was injected intramuscularly with penicillin. At necropsy most female pigs were pregnant.

\section{DISCUSSION}

\section{PARASITOLOGY}

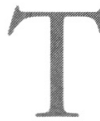
There was a tendency towards a decrease in prevalence of $T$. suis positive pigs with increasing inoculation dose. This could be due to a more vigorous stimulation of the immune system in those pigs receiving a high inoculation dose (Keymer, 1982). Likewise there was an indication of a decline in establishment associated with an increase in numbers of eggs given. This has formerly been described in single infections (Hale \& Stewart, 1979) and trickle infections (Powers et al., 1960) and could reflect a self-regulation of the T. suis population size. A prevalence of 15-42\% T. suis positive pigs has been reported in naturally infected pigs which harboured a mean number of 629 worms (Jacobs \& Dunn, 1969; Pattison et al., 1980). In the low dose group worms were located mainly in the caecum and the upper colon. With increasing dose worms were also found in the more aboral parts of the colon, which was most pronounced in the high dose group. Trichuris suis is reported to have a predilection for the caecum and the upper third of the colon, but the entire colon is a potential habitat, with worms being found occasionally in the rectum (Powers et al., 1960). A similar phenomenon has been described in pigs inoculated with increasing doses of Oesophagostomum spp. larvae (Christensen et al., 1995).

The lengths of adult $T$. suis recorded in this study were less $(27-36 \mathrm{~mm})$ than those $(48-50 \mathrm{~mm})$ reported by Beer (1973b). This is likely to be due to differences in parasite strains and the fact that $T$. suis worms continue to grow after reaching the adult stage. However, preservation of worms in iodine may cause minor shrinkage. Worms recovered from pigs receiving a high inoculation dose were shorter than those from the low and medium dose groups, which has also been reported for pigs inoculated with different dose levels of Oesophagostomum larvae (Christensen et al., 1995). This suggests a crowding effect with suboptimal growth conditions, a view that is supported by the enlargement of the intestinal habitat. The observed proportion of female worms was quite constant in the three inoculated groups (i.e. 0.41-0.43) and probably represents a level characteristic of established adult T. suis populations in experimentally infected pigs, although it may reflect the sex proportions of the infective larvated eggs. For comparison Jacobs \& Dunn (1969) found that the proportion of female T. suis was $0.55-$ 0.66 in naturally infected pigs.

Our study shows that T. suis has an aggregated distribution within pig populations which becomes more pronounced with increasing inoculation dose. This overdispersion, which implies that a few hosts harbour most worms while the majority of hosts have few or no worms, has also been documented for T. trichiura in human populations (Bundy \& Cooper, 1989) and is of major importance in understanding the epidemiology and control of this parasite (Keymer, 1982).

The observed rise in mean egg production followed by a decline has been described to be typical of $T$. suis (Powers et al., 1960). In the low dose group faecal egg excretion was quite constant as reflected by the similar course and culmination of the median and mean e.p.g. curves. The medium dose group had consistently higher median and mean e.p.g. levels compared to the low dose group except for wk 12 p.i.; the somewhat earlier culmination of the median compared to the mean suggests a decrease in prevalence of egg-excreting pigs in the weeks following patency. In the high dose group the median e.p.g. was very low during the entire patent period possibly due to expulsion of larval infection in a number of pigs while the high mean e.p.g. level largely represents a few heavily infected pigs. In order to establish a moderate infection in a high proportion of experimentally infected pigs the medium dose seems to be preferable: except for wk 12 p.i. the "average" (median) pig had the highest egg excretion in the medium group followed by the low 
dose group whereas the lowest value was found in the high dose group. This tendency has also been reported following trickle infections with oesophagostomes in pigs (Roepstorff et al., 1996). Assuming a daily faecal excretion amounting to two kg this would correspond to $19,000(6,800-320,000), 800(0-12,000)$ and 18,200 (17,200-19,000) eggs (median, (min.-max.)) produced per female per day in the low, medium and high dose group, respectively, indicating that $T$. suis is not necessarily such a non-prolific egg layer as suggested by Beer et al. (1971).

The unintentional infection of two control pigs is likely to be due to infective eggs passing unhatched through the intestinal tract of inoculated pigs and subsequently causing infection in pigs that ingest faeces (Hill, 1973). The observed decrease in faecal egg output after wk 9 p.i. indicates that there was an ongoing elimination of $T$. suis taking place in the weeks preceding slaughter. This is in line with the finding that pigs single infected with $A$. suum started to eliminate worms earlier and to a larger extent with increasing inoculation dose (Roepstorff et al., 1997).

\section{HAEMATOlOgy \& ANimAL PERFORMANCE}

A loss of albumin is reported to occur as T. suis larvae re-emerge to establish as adults on the mucosal surface of the intestine (Sansom et al., 1974; Batte et al., 1977). This is confirmed in the present study by the steeper fall in serum albumin levels in the high dose group from wk 2-6 p.i. compared to the other groups. Batte et al. (1977) observed that a marked decrease in the albumin fraction of the total serum protein occurred beginning seven to 14 days p.i.

Anaemia was not observed in any group as deemed by PCV and haemoglobin levels. An average rate of loss of erythrocytes which was approximately proportional to the infective dose of eggs ranging from seven to $35 \mathrm{ml} /$ day has been reported by Beer et al. (1974).

The lack of peripheral eosinophilia due to $T$. suis infection seen in the present study has been reported formerly (Beer \& Lean, 1973), although eosinophils have been described to be prominent in the colonic mucosa of infected pigs (Powers et al., 1960).

Clinical manifestations of experimental trichuriosis may follow from inoculation with 25,000 to 400,000 infective eggs (Powers et al., 1960; Batte \& Moncol, 1972; Beer \& Lean, 1973; Beer et al., 1973; Batte et al., 1977). On the other hand some authors registered neglible clinical signs and haematological changes following inoculation with 15,000 eggs (Beer et al., 1971; Beer et al., 1974). Apart from dose size the main determinant for the presence of clinical signs seems to be whether or not secondary microbial invasion occurs (Beer, 1973b; Mansfield \& Urban, 1996) although there are most likely also differences in parasite strains.
Symptoms seem to coincide with the emergence of the larvae from their histotrophic phase of development to form 3rd and 4th stage larvae (Batte et al., 1977). In this study body weights in the groups were comparable. However, pigs in this experiment were heavier at the time of inoculation, which makes direct comparison with other studies reporting decreases in weight gains difficult (Beer et al., 1973; Hale \& Stewart, 1979).

The results of the current study suggest that dose level influences the course of experimental $T$. suis infection in pigs in terms of parasitological parameters i.e. there exists a dynamic regulation of the adult T. suis burden, which could have important implications for the comparison of epidemiology and control of T. trichiura infection in humans. We observed an increase in worm aggregation with increasing dose, suggesting that persons with high worm burdens in a heavily contaminated environment might be especially important for the propagation and potential control measures of this parasitic infection.

\section{ACKNOWLEDGEMENT}

T The help of Karen Røndal Lauritsen is gratefully appreciated. The Danish National Research Foundation is acknowledged for financial support. This experiment complies with the current laws of Denmark.

\section{REFERENCES}

BatTe E.G. \& Moncol D.J. Whipworms and dysentery in feeder pigs. Journal of the American Veterinary Medical Association, 1972, 161, 1226-1228.

Batte E.G., Mclamb R.D., Muse K.E., Tally S.D. \& Vestal T.J. Pathophysiology of swine trichuriasis. American Journal of Veterinary Research, 1977, 38, 1075-1079.

BEER R.J.S. Studies on the biology of the life-cycle of Trichuris suis Schrank, 1788. Parasitology, 1973a, 67, 253-262.

BEER R.J.S. Morphological descriptions of the egg and larval stages of Trichuris suis Schrank, 1788. Parasitology, 1973b, 67, 263-278.

BEER R.J.S. \& LEAN I.J. Clinical trichuriasis produced experimentally in growing pigs. Part I: Pathology of infection. Veterinary Record, 1973, 93, 189-195.

BeER R.J., TafFs L.F., Jacobs D.E., Lean I.J. \& Curran M.K. Evaluation of dichlorvos (v3 formulation) against larval and adult Trichuris suis and observations on experimental infection in growing pigs. Veterinary Record, 1971, 88, 436441.

BeEr R.J.S., Lean I.J., Jacobs D.E. \& Curran M.K. Clinical trichuriasis produced experimentally in growing pigs. Part II: Anthelmintic efficacy of dichlorvos. Veterinary Record, 1973, 93, 195-197. 
BeER R.J., SANsom B.F. \& Taylor P.J. Erythrocyte losses from pigs with experimental Trichuris suis infections measured with a whole-body counter. Journal of Comparative Pathology, 1974, 84, 331-346.

BIEHL L.G. Internal parasitism of feeder pigs in Southern Illinois. Agri-practice, 1984, 5, 20-26.

Bundy D.A.P. \& COOPER E.S. Trichuris and trichuriasis in humans. Advances in Parasitology, 1989, 28, 107-173.

BuRden D.J. \& HAMmET N.C. A comparison of the infectivity of Trichuris suis ova embryonated by four different methods. Veterinary Parasitology, 1976, 2, 307-311.

Burden D.J. \& Hammet N.C. The development and survival of Trichuris suis ova on pasture plots in the south of England. Research in Veterinary Science, 1979, 26, 66-70.

Burden D.J., Hammet N.C. \& Brookes P.A. Field observations on the longevity of Trichuris suis ova. Veterinary Record, 1987, 121, 43.

Christensen C.M., Barnes E.H., Nansen P., Roepstorff A. \& Slotved H.-C. Experimental Oesophagostomum dentatum infection in the pig: worm popula tions resulting from single infections with three doses of larvae. International Journal for Parasitology, 1995, 25, 1491-1498.

HALE O.M. \& STEWART T.B. Influence of an experimental infection of Trichuris suis on performance of pigs. Journal of Animal Science, 1979, 49, 1000-1005.

HiLl C.H. The survival of swine whipworm eggs in hog lots. Journal of Parasitology, 1957, 43, 104.

HiLL C.H. Infectivity of embryonated T. suis eggs passed through the digestive tracts of post-weanling pigs. Journal of Parasitology, 1973, 59, 580-581.

Holland C. Neglected infections-trichuriasis and strongyloidiasis, in: Impact of Helminth Infections on Human Nutrition. Stephenson, L.S. (ed), Taylor \& Francis, London, New York \& Philadelphia, 1987, 161-201.

JACOBS D.E. \& DunN A.M. Helminths of Scottish pigs: occurrence, age incidences and seasonal variations. Journal of Helminthology, 1969, 43, 327-340.

JENKINS T. A morphological and histochemical study of Trichuris suis (Schrank, 1788) with special reference to the host-parasite relationship. Parasitology, 1970, 61, 357-374.

Johansen M.V., Bøgh H.O., Giver H., Eriksen L., Nansen P., STEPHENSON L.S. \& KNUdSEN K.E.B. Schistosoma japonicum and Trichuris suis infections in pigs fed diets with high and low protein. Parasitology, 1997, 115, 257-264.

Keymer A. Density-dependent mechanisms in the regulation of intestinal helminth populations. Parasitology, 1982, 84, 573-587.

Mansfield L.S. \& URBAN Jr J.F. The pathogenesis of necrotic proliferative colitis in swine is linked to whipworm induced suppression of mucosal immunity to resident bacteria. Veterinary Immunology and Immunopatbology, 1996, 50, 1-17.

Miller E.R. \& UllRey D.E. The pig as a model for human nutrition. Annual Reviews of Nutrition, 1987, 7, 361-382.

Pattison H.D., Thomas R.J. \& Smith W.C. A survey of gastrointestinal parasitism in pigs. Veterinary Record, 1980, 107, 415-418.
Powers K.G., TodD A.C. \& GoldsBy A.I. Swine whipworm in Wisconsin. Veterinary Medicine, 1959, 54, 396-397.

Powers K.G., Todd A.C. \& McNutT S.H. Experimental infections of swine with Trichuris suis. American Journal of Veterinary Research, 1960, 21, 262-268.

ROEPSTORFF A. \& MurRell K.D. Transmission dynamics of helminth parasites of pigs on continuous pasture: Ascaris suum and Trichuris suis. International Journal for Parasitology, 1997, 27, 563-572.

RoEPSTORFF A. \& NANSEN P. The epidemiology, diagnosis and control of helminth parasites of swine. A FAO animal health manual, 1999. FAO, Rome, Italy.

Roepstorff A., Bjørn H., Nansen P., Barnes E.H. \& ChrisTENSEN C.M. Experimental Oesophagostomum dentatum infections in the pig: worm populations resulting from trickle infections with three dose levels of larvae. International Journal for Parasitology, 1996, 26, 399-408.

Roepstorff A., ERiksen L., Slotved H.-C. \& NANSEn P. Experimental Ascaris suum infection in the pig: worm population kinetics following single inoculations with three doses of infective eggs. Parasitology, 1997, 115, 443-452.

Sansom B.F., BeEr R.J.S. \& Kitchenham B.A. Changes in the concentrations of serum urea nitrogen, albumin, globulin, sodium, and inorganic phosphorus in weaner pigs infected with Trichuris suis. Journal of Comparative Pathology, 1974, 84, 409-415.

STEPHENSON L.S. Impact of helminth infections on human nutrition. Taylor \& Francis; London, New York \& Philadelphia, 1987.

Reçu le 22 mai 2000 Accepté le 21 août 2000 